\title{
Multibody charmless b-hadron decays
}

\author{
Giulio Dujany ${ }^{* \dagger}$ \\ University of Manchester \\ E-mail: giulio.dujanyecern.ch
}

Multibody charmless b-hadron decays are a unique laboratory to probe $\mathrm{CP}$ violation, to look for effects of new physics and to provide insights into the hadronisation mechanism. The decay modes $\Lambda_{b}^{0} \rightarrow \Lambda K^{+} K^{-}, \Lambda_{b}^{0} \rightarrow \Lambda K^{+} \pi^{-}, \Lambda_{b}^{0} \rightarrow \Lambda \phi$ and $B_{s}^{0} \rightarrow K_{\mathrm{S}}^{0} K^{*}$ are observed for the first time by LHCb and their branching fractions are measured. Evidence is seen for the $\Lambda_{b}^{0} \rightarrow \Lambda \pi^{+} \pi^{-}$decay and limits are set for the decays $\Xi_{b}^{0} \rightarrow \Lambda h^{+} h^{(\prime)}$ and $B^{0} \rightarrow K_{\mathrm{S}}^{0} K^{*}$. The phase-space integrated $\mathrm{CP}$ asymmetry parameters are measured for $\Lambda_{b}^{0} \rightarrow \Lambda K^{+} K^{-}$and $\Lambda_{b}^{0} \rightarrow \Lambda K^{+} \pi^{-}$while several triple-product asymmetries are measured for $\Lambda_{b}^{0} \rightarrow \Lambda \phi$. All these asymmetries are found to be compatible with zero.

16th International Conference on B-Physics at Frontier Machines

2-6 May 2016

Marseille, France

* Speaker.

${ }^{\dagger}$ On behalf of the LHCb collaboration. 


\section{Introduction}

Multibody charmless b-hadron decays are a unique laboratory to probe $\mathrm{CP}$ violation and to look for effects of new physics. Charmless decays often proceed via a $b \rightarrow u$ transition, dominated by tree-level Feynman diagrams, and $b \rightarrow s$ and $b \rightarrow d$ transitions, dominated by penguin loop diagrams. Due to the small magnitude of the $V_{u b}$ Cabibbo-Kobyashi-Maskawa matrix element, tree and penguin diagrams can have similar magnitude. As there is a relative weak phase - in the Standard Model (SM) the CKM angle $\gamma$ - their interference can give rise to CP violation in decay. Moreover beyond the SM particles could produce sizeable differences with respect to the SM expectations both in the branching fractions and in the amount of $\mathrm{CP}$ violation, by entering as virtual particles in the loops of the penguin diagrams.

Multibody decays can proceed through intermediate states and the paths from the initial to the final state interfere. Moreover different regions of the phase space can present different levels of $\mathrm{CP}$ violation. As an example, Figure 1 shows the raw asymmetry, which is the sum of the $\mathrm{CP}$ asymmetry, the production and the detection asymmetry, in $B^{+} \rightarrow K^{+} \pi^{+} \pi^{-}$where large variations are evident and the local CP asymmetries are as large as 60\% [3].

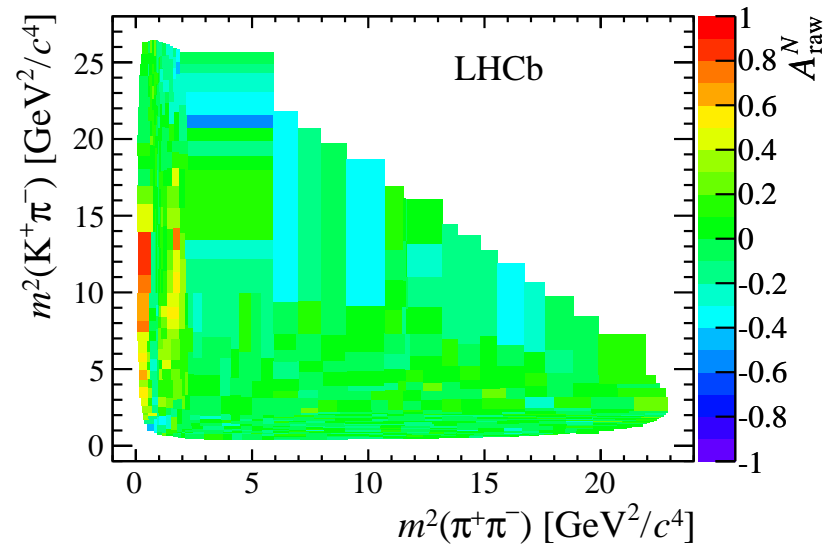

Figure 1: Measured raw asymmetry in Dalitz plot bins of background-subtracted and acceptance-corrected events for $B^{+} \rightarrow K^{+} \pi^{+} \pi^{-}$decays.

The LHCb detector [1] is a single-arm forward spectrometer covering the pseudorapidity range $2<\eta<5$, designed for the study of particles containing $b$ or $c$ quarks. The detector elements that are particularly relevant for the analyses described in these proceedings are: a silicon-strip vertex detector surrounding the $p p$ interaction region that allows $c$ - and $b$-hadrons to be identified from their characteristically long flight distance; a tracking system that provides a measurement of the momentum of charged particles; and two ring-imaging Cherenkov detectors that are able to discriminate between different species of charged hadrons. During Run I, LHCb collected an integrated luminosity of approximately $3 \mathrm{fb}^{-1}$, of which $1 \mathrm{fb}^{-1}$ was gathered at $\sqrt{s}=7 \mathrm{TeV}$ and $2 \mathrm{fb}^{-1}$ at $\sqrt{s}=8 \mathrm{TeV}$.

The $K_{\mathrm{S}}^{0} \rightarrow \pi^{+} \pi^{-}$decay can be reconstructed in two different categories at LHCb: the first involving $K_{\mathrm{S}}^{0}$ mesons that decay early enough for the daughter pions to be reconstructed in the vertex detector, and the second containing $K_{\mathrm{S}}^{0}$ mesons that decay later such that track segments of the pions cannot be formed in the vertex detector. These categories are referred to as long and downstream, respectively. The long category has better mass, momentum and vertex resolution than the downstream category. Similar categories are used for the $\Lambda \rightarrow p \pi^{-}$decay. 
Three recent results from $\mathrm{LHCb}$ in the field of charmless multibody b-hadron decays are here reviewed.

2. $\Lambda_{b}^{0}\left(\Xi_{b}^{0}\right) \rightarrow \Lambda h^{+} h^{(\prime)-}$

Among the possible charmless hadronic final states of $b$ baryons, only the two-body $\Lambda_{b}^{0} \rightarrow$ $p K^{-}$and $\Lambda_{b}^{0} \rightarrow p \pi^{-}$decays [4] and the three-body $\Lambda_{b}^{0} \rightarrow K_{\mathrm{S}}^{0} p \pi^{-}$decay [5] have been observed, while evidence has been reported for the $\Lambda_{b}^{0} \rightarrow \Lambda \eta$ decay [6]. No decay of a $\Xi_{b}^{0}$ baryon to a charmless final state has yet been observed. The class of decays $\Lambda_{b}^{0}\left(\Xi_{b}^{0}\right) \rightarrow \Lambda h^{+} h^{(\prime)}$ ( where $h$ is a pion or a kaon) is of great interest because they can present potentially large CP violation effects and because they provide insights into the underlying dynamical mechanisms in $b$ baryon decays.

A search for these decays is performed with the full LHCb Run I dataset [7]. Each $b$-baryon candidate is reconstructed by combining two oppositely charged tracks with a $\Lambda$ candidate, where the $\Lambda$ candidates are reconstructed by combining $p$ and $\pi^{-}$tracks. Loose particle identification criteria (PID) are required for the $p$ track to reduce backgrounds from $K_{\mathrm{S}}^{0}$ decays. The dataset is then separated into the different final states under study applying further particle identification criteria on the di-hadron pair. A boosted decision tree (BDT) classifier, trained on sideband background and simulated signal events, is used to further suppress the combinatorial background. The decay $\Lambda_{b}^{0} \rightarrow \Lambda_{c}^{+}\left(\rightarrow \Lambda \pi^{+}\right) \pi^{-}$is taken as normalisation mode. Six different categories are considered corresponding to three data taking periods and to the two reconstruction categories of the $\Lambda$.

The yields of all signals as well as that of the normalisation mode are extracted with a simultaneous unbinned maximum likelihood fit to the six different categories. By a simultaneous fit, the common shape parameters among the different categories can be shared and the cross-feed background ${ }^{1}$ from the different final states is better constrained. Contributions from a smoothly varying combinatorial background and from the partially reconstructed backgrounds, where a photon or a neutral pion are not reconstructed in a decay of a real $b$ baryon, are also modelled. The regions of the $\Lambda h^{+} h^{(\prime)}$ - invariant mass spectra corresponding to the $\Lambda_{b}^{0}$ and $\Xi_{b}^{0}$ invariant masses were not inspected until the candidate selection and the fit model were finalised, in order to avoid any bias. The result of the fit is shown in Figure 2. Signals for $\Lambda_{b}^{0} \rightarrow \Lambda K^{+} K^{-}$and $\Lambda_{b}^{0} \rightarrow \Lambda K^{+} \pi^{-}$are observed with a significance of $15.8 \sigma$ and $8.1 \sigma$, respectively, where the systematic uncertainties are also taken into account. There is strong evidence for $\Lambda_{b}^{0} \rightarrow \Lambda \pi^{+} \pi^{-}(4.7 \sigma)$ while the significance of the other modes is less than $3 \sigma$. Branching fraction central values and confidence intervals are obtained by combining the individual branching fractions for each of the six categories, taking systematic uncertainties into account. The results for the absolute branching fractions are

$$
\begin{aligned}
\mathscr{B}\left(\Lambda_{b}^{0} \rightarrow \Lambda K^{+} \pi^{-}\right) & =(5.6 \pm 0.8 \pm 0.8 \pm 0.7) \times 10^{-6}, \\
\mathscr{B}\left(\Lambda_{b}^{0} \rightarrow \Lambda K^{+} K^{-}\right) & =(15.9 \pm 1.2 \pm 1.2 \pm 2.0) \times 10^{-6}, \\
\mathscr{B}\left(\Lambda_{b}^{0} \rightarrow \Lambda \pi^{+} \pi^{-}\right) & =(4.6 \pm 1.2 \pm 1.4 \pm 0.6) \times 10^{-6},
\end{aligned}
$$

where the first uncertainty is statistical, the second systematic and the third one is due to the precision with which the normalisation channel branching fraction is known. Since no evidence is seen for the $\Xi_{b}^{0}$ decays, upper limits are calculated using a Bayesian prescription, with a prior removing

\footnotetext{
${ }^{1}$ Background coming from $K \leftrightarrow \pi$ misidentification such that the signal in one of the final states is a background in another.
} 

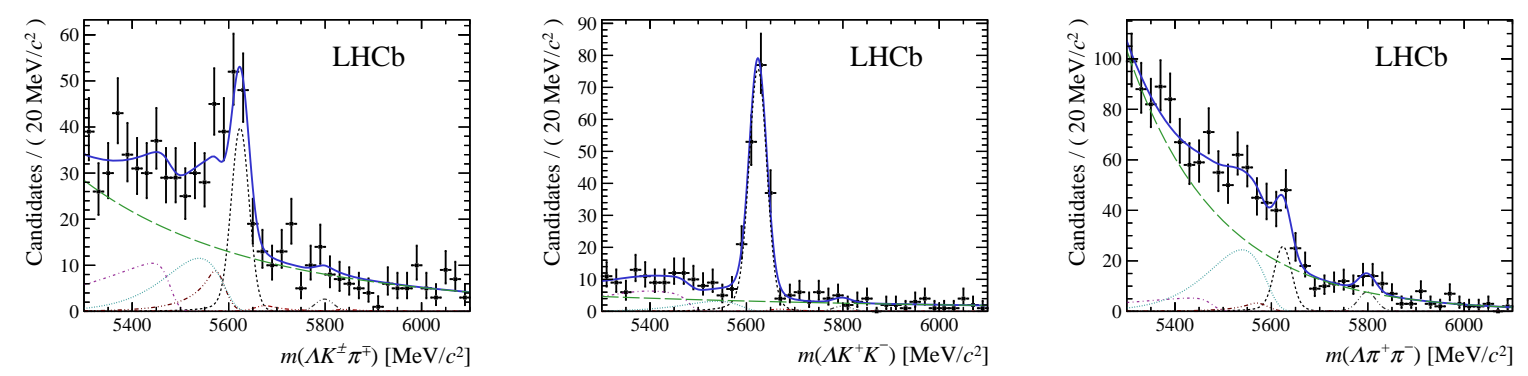

Figure 2: Results of the fit for the (left) $\Lambda K^{ \pm} \pi^{\mp}$, (middle) $\Lambda K^{+} K^{-}$and (right) $\Lambda \pi^{+} \pi^{-}$final states for all categories combined. Superimposed on the data are the total result of the fit as a solid blue line, the $\Lambda_{b}^{0}\left(\Xi_{b}^{0}\right)$ signal contribution as a short-dashed black (double dot-dashed grey) line, cross-feed as triple dotdashed brown lines, the combinatorial background as a long-dashed green line, and partially reconstructed background with either a missing neutral pion as a dot-dashed purple line or a missing soft photon as a dotted cyan line.

the unphysical region

$$
\begin{aligned}
& f_{\Xi_{b}^{0}} / f_{\Lambda_{b}^{0}} \times \mathscr{B}\left(\Xi_{b}^{0} \rightarrow \Lambda \pi^{+} \pi^{-}\right)<1.7(2.1) \times 10^{-6} \text { at } 90(95) \% \mathrm{CL}, \\
& f_{\Xi_{b}^{0}} / f_{\Lambda_{b}^{0}} \times \mathscr{B}\left(\Xi_{b}^{0} \rightarrow \Lambda K^{-} \pi^{+}\right)<0.8(1.0) \times 10^{-6} \text { at } 90(95) \% \mathrm{CL}, \\
& f_{\Xi_{b}^{0}} / f_{\Lambda_{b}^{0}} \times \mathscr{B}\left(\Xi_{b}^{0} \rightarrow \Lambda K^{+} K^{-}\right)<0.3(0.4) \times 10^{-6} \text { at } 90(95) \% \mathrm{CL},
\end{aligned}
$$

where a factor corresponding to the currently unknown ratio of the $\Xi_{b}^{0}$ and $\Lambda_{b}^{0}$ production fraction is included.

The phase-space integrated CP asymmetry is measured for $\Lambda_{b}^{0} \rightarrow \Lambda K^{+} K^{-}$and $\Lambda_{b}^{0} \rightarrow \Lambda K^{+} \pi^{-}$ decays which present significant signal yields. The simultaneous extended maximum likelihood fit is modified to allow the determination of the raw asymmetry defined as

$$
\mathscr{A}_{C P}^{\text {raw }}=\frac{N_{f}^{\text {corr }}-N_{\bar{f}}^{\text {corr }}}{N_{f}^{\text {corr }}+N_{\bar{f}}^{\text {corr }}},
$$

where $N_{f}^{\text {corr }}\left(N_{\bar{f}}^{\text {corr }}\right)$ is the efficiency-corrected yields for $\Lambda_{b}^{0}\left(\bar{\Lambda}_{b}^{0}\right)$. To measure the underlying CP violation, the raw asymmetry has to be corrected for possible small detection $\left(\mathscr{A}_{D}\right)$ and production $\left(\mathscr{A}_{P}\right)$ asymmetries, $\mathscr{A}_{C P}=\mathscr{A}_{\text {raw }}-\mathscr{A}_{D}-\mathscr{A}_{P}$. This can be achieved with the $\Lambda_{b}^{0} \rightarrow \Lambda_{c}^{+}\left(\rightarrow \Lambda \pi^{+}\right) \pi^{-}$ control mode, which is expected to have negligible CP violation. Since this mode shares the same initial state as the decay of interest, it has the same production asymmetry; moreover, the final-state selection differs only in the PID requirements and therefore most detection asymmetry effects also cancel. The results for the phase-space integrated $\mathrm{CP}$ asymmetries are

$$
\begin{aligned}
& \mathscr{A}_{C P}\left(\Lambda_{b}^{0} \rightarrow \Lambda K^{+} \pi^{-}\right)=-0.53 \pm 0.23 \pm 0.11, \\
& \mathscr{A}_{C P}\left(\Lambda_{b}^{0} \rightarrow \Lambda K^{+} K^{-}\right)=-0.28 \pm 0.10 \pm 0.07,
\end{aligned}
$$

where the uncertainties are statistical and systematic, respectively. These are both less than $3 \sigma$ from zero, indicating consistency with CP symmetry.

3. $\Lambda_{b}^{0} \rightarrow \Lambda \phi$

In the SM the decay $\Lambda_{b}^{0} \rightarrow \Lambda \phi$ proceeds via a $b \rightarrow s \bar{s} s$ penguin transition and the presence of 
beyond-the-SM particles in the loop could induce $\mathrm{CP}$ violation effects beyond those expected in the SM. The first observation of this decay mode is performed with the full LHCb Run I dataset and the CP asymmetry is studied by means of $T$-odd observables [8]. The decay $B^{0} \rightarrow K_{\mathrm{S}}^{0} \phi$ is used as normalisation mode. Each $\Lambda_{b}^{0}\left(B^{0}\right)$ candidate is reconstructed by combining a $\Lambda$ from $\Lambda \rightarrow p \pi^{-}\left(K_{\mathrm{S}}^{0}\right.$ from $\left.K_{\mathrm{S}}^{0} \rightarrow \pi^{+} \pi^{-}\right)$and a $\phi$ from $\phi \rightarrow K^{+} K^{-}$. Two different categories are used depending on whether the $\Lambda$ or $K_{\mathrm{S}}^{0}$ are reconstructed as long or downstream. Different BDTs are used for reducing the combinatorial background in the two categories. Those BDTs are trained using simulated events as signal and sideband from data as background. To extract the signal yield a three-dimensional unbinned maximum likelihood fit in $m_{K^{+} K^{-}} p \pi^{-}, m_{K^{+} K^{-}}$and $m_{p \pi^{-}}$is used. Along with the signal, different backgrounds are considered: $\Lambda_{b}^{0} \rightarrow \Lambda K^{+} K^{-}$non-resonant, $\phi+\Lambda$ and pure combinatorial. In order to avoid any bias in the analysis the regions of the $\Lambda K^{+} K^{-}$invariant mass spectra corresponding to the $\Lambda_{b}^{0}$ invariant mass were not inspected until the candidate selection and fit model were finalised. The result of the fit for the long category is shown in Figure 3. The signal
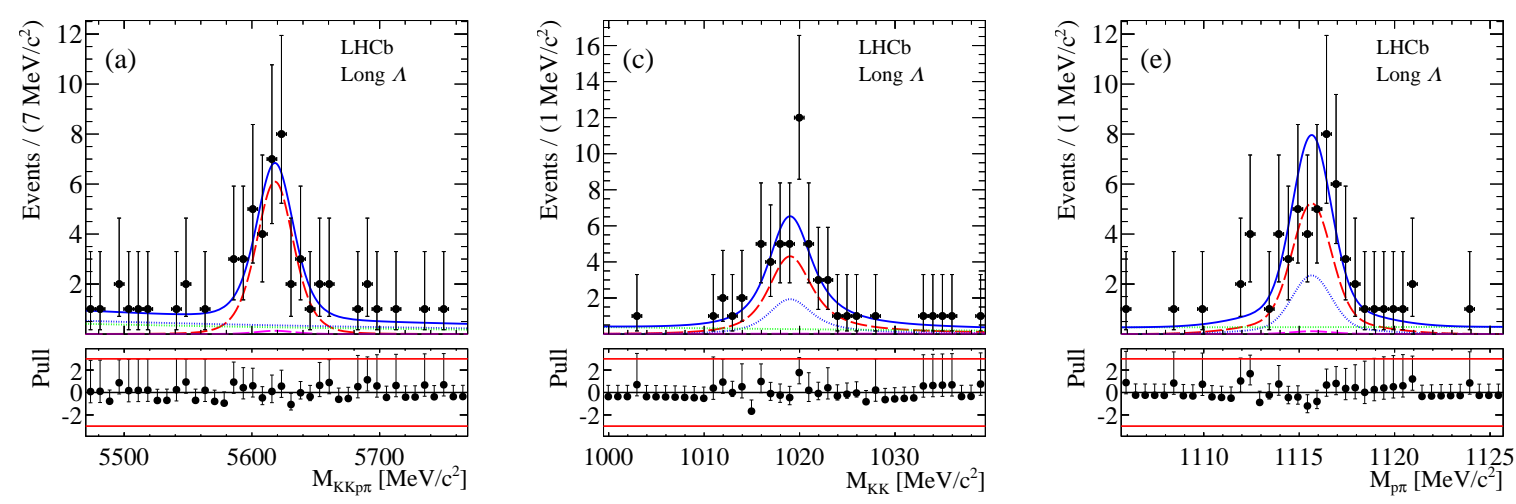

Figure 3: Fit projections to the (left) $p \pi^{-} K^{+} K^{-}$invariant mass, (middle) $K^{+} K^{-}$invariant mass, and (right) the $p \pi^{-}$invariant mass for the long category. The total fit projection is given by the blue solid line. The blue and green dotted lines represent the $\phi+\Lambda$ and pure combinatorial fit components, respectively. The red and magenta dashed lines represent the $\Lambda_{b}^{0} \rightarrow \Lambda \phi$ signal and the $\Lambda_{b}^{0} \rightarrow \Lambda K^{+} K^{-}$non-resonant components, respectively. Black points represent the data. Data uncertainties are Poisson $68 \%$ confidence intervals.

is observed with a significance of $5.9 \sigma$ once the systematic uncertainties are taken into account. The branching fraction is evaluated to be

$$
\mathscr{B}\left(\Lambda_{b}^{0} \rightarrow \Lambda \phi\right)=\left(5.15 \pm 1.04 \pm 0.35_{-0.43}^{+0.50} \pm 0.44\right) \times 10^{-6},
$$

where the first uncertainty is statistical, the second systematic, the third comes from the precision on the normalisation mode and the last from the precision on $f_{d} / f_{\Lambda_{b}^{0}}[9]$.

As the $\Lambda_{b}^{0}$ could be produced polarised, five angles are needed to describe this decay. The sine and the cosine of the angle $\Phi_{1}\left(\Phi_{2}\right)$ between the orthogonal direction to the decay plane of $\Lambda(\phi)$ and the direction orthogonal to the beam and the direction of flight of the $\Lambda_{b}^{0}$ are $T$-odd observables [10]. It is thus possible to define asymmetries in $\cos \Phi_{i}$ and $\sin \Phi_{i}$

$$
A_{i}^{c / s}=\frac{N_{i}^{+c / s}-N_{i}^{-c / s}}{N_{i}^{+c / s}+N_{i}^{-c / s}},
$$

where, for example, $N_{i}^{+c}$ is the number of $\Lambda_{b}^{0}$ with $\cos \Phi_{i}>0$. These asymmetries are determined experimentally through a simultaneous unbinned maximum likelihood fit to different datasets in 
which the relevant observables are either positive or negative and in which the yields are parameterised in terms of the total yield and the asymmetry. The determined asymmetries are

$$
\begin{array}{ll}
A_{\Lambda}^{c}=-0.22 \pm 0.12 \pm 0.06, & A_{\Lambda}^{s}=0.13 \pm 0.12 \pm 0.05, \\
A_{\phi}^{c}=-0.01 \pm 0.12 \pm 0.03, & A_{\phi}^{s}=-0.07 \pm 0.12 \pm 0.01,
\end{array}
$$

where the first uncertainty is statistical and the second systematic. The values are compatible with zero.

4. $B_{(s)}^{0} \rightarrow K_{\mathrm{S}}^{0} K^{*}$

A search for the decays $B_{(s)}^{0} \rightarrow K_{\mathrm{S}}^{0} K^{*}$ is performed with $1 \mathrm{fb}^{-1}$ at $\sqrt{s}=7 \mathrm{TeV}$ using as normalisation mode $B^{0} \rightarrow K_{\mathrm{S}}^{0} \pi^{+} \pi^{-}$to determine the branching fractions [11]. Each signal $B_{(s)}^{0}$ candidate is obtained by combining a $K_{\mathrm{S}}^{0}$ from $K_{\mathrm{S}}^{0} \rightarrow \pi^{+} \pi^{-}$with a $K^{* 0}$ from $K^{* 0} \rightarrow K^{+} \pi^{-}$. Two different categories are used depending on whether the $K_{\mathrm{S}}^{0}$ are reconstructed as long or downstream. The selection makes use of particle identification variables to discriminate between protons, pions and kaons and of a BDT to reduce the combinatorial background. Candidates are excluded if the reconstructed mass of a two-body intermediate state is within $30 \mathrm{MeV}(48 \mathrm{MeV})$ of the known mass of the relevant charm (charmonium) resonance. The signal yield is extracted with a two-dimensional unbinned maximum likelihood fit in $m_{K_{\mathrm{S}}^{0} K^{ \pm} \pi^{\mp}}$ and $m_{K^{ \pm} \pi^{\mp}}$ while the yield of the normalisation mode is obtained with an unbinned maximum likelihood fit in $m_{K_{S}^{0} \pi^{+} \pi^{-}}$. To avoid any bias in the analysis, the signal region was not inspected until the selection and fit procedure were finalised. Figure 4
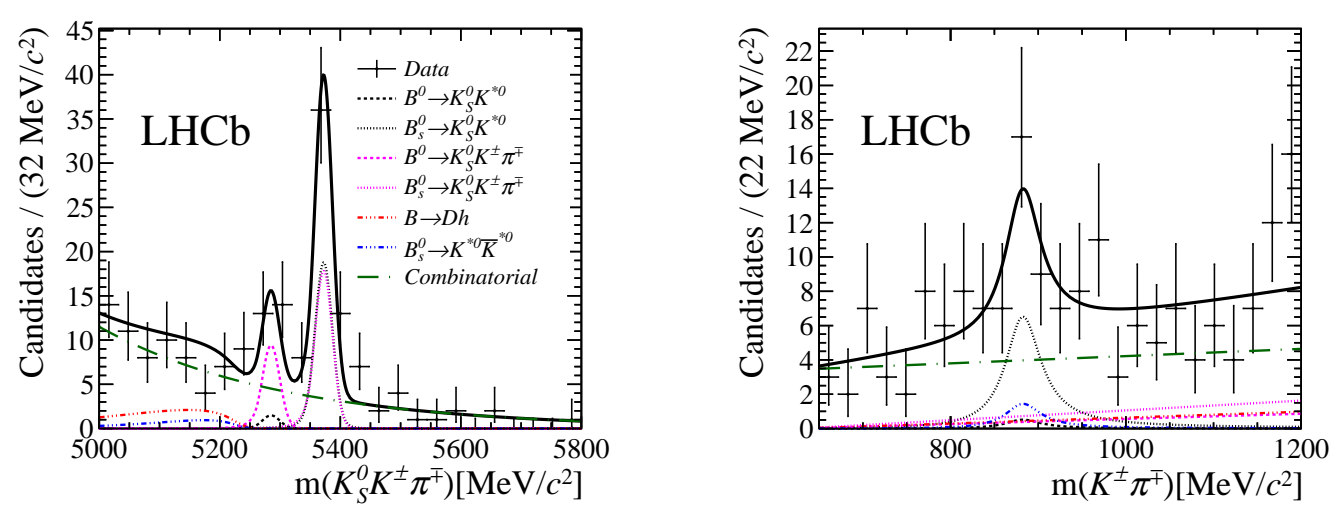

Figure 4: Distribution of (left) $K_{s}^{0} K^{ \pm} \pi^{\mp}$ mass and (right) $K^{ \pm} \pi^{\mp}$ mass with fit results overlaid for the downstream category. The data are shown as black points with error bars. The overall fit is represented by the solid black line. The $B^{0}$ and $B_{s}^{0}$ signal components are the black short-dashed and dotted lines respectively, while the non-resonant components are the magenta short-dashed and dotted lines. The partially reconstructed backgrounds are the red triple-dotted line $(B \rightarrow D h)$ and the blue triple-dotted line $\left(B_{s}^{0} \rightarrow\right.$ $\left.K^{* 0} K^{* 0}\right)$. The combinatorial background is the green long-dash dotted line.

shows the result of the fit for the downstream category. The decay $B_{s}^{0} \rightarrow K_{\mathrm{S}}^{0} K^{*}$ is observed with a significance of $7.1 \sigma$ taking into account also the systematic uncertainties; its branching fraction is measured to be

$$
\left.\mathscr{B}\left({\overline{B_{s}}}^{\prime}\right) \rightarrow K_{s}^{0} K^{*}\right)=(16.4 \pm 3.4 \pm 1.9 \pm 1.0 \pm 0.7) \times 10^{-6},
$$


where the first uncertainty is statistical, the second systematic, the third is from the precision on $f_{s} / f_{d}$ and the last is from the precision on the branching fraction of the normalisation mode. No significant signal is observed for $B^{0} \rightarrow K_{\mathrm{S}}^{0} K^{*}$ and an upper limit on its branching fraction is set to be

$$
\mathscr{B}\left((\bar{B}) \rightarrow K_{s}^{0} K^{*}\right)<0.96(1.04) \times 10^{-6} \text { at } 90 \%(95 \%) \mathrm{CL}
$$

\section{Conclusions}

Three recent results from $\mathrm{LHCb}$ in the field of multibody charmless $b$-decays have been presented. The decay modes $\Lambda_{b}^{0} \rightarrow \Lambda K^{+} K^{-}, \Lambda_{b}^{0} \rightarrow \Lambda K^{+} \pi^{-}, \Lambda_{b}^{0} \rightarrow \Lambda \phi$ and $B_{s}^{0} \rightarrow K_{\mathrm{S}}^{0} K^{*}$ are observed for the first time and their branching fractions are measured. Evidence is seen for the $\Lambda_{b}^{0} \rightarrow \Lambda \pi^{+} \pi^{-}$ decay and limits are set for the decays $\Xi_{b}^{0} \rightarrow \Lambda h^{+} h^{(\prime)-}$ and $B^{0} \rightarrow K_{\mathrm{S}}^{0} K^{*}$. The phase-space integrated $\mathrm{CP}$ asymmetry parameters are measured for the $\Lambda_{b}^{0} \rightarrow \Lambda K^{+} K^{-}$and $\Lambda_{b}^{0} \rightarrow \Lambda K^{+} \pi^{-}$decays, while several triple-product asymmetries are measured for the $\Lambda_{b}^{0} \rightarrow \Lambda \phi$ decay. All these asymmetries are found to be compatible with zero.

\section{References}

[1] Alves Jr. A. A. et al. (LHCb collaboration), The LHCb detector at the LHC, JINST 3 S08005 (2008).

[2] Beringer J. et al. (Particle Data Group), Review of Particle Physics, Phys.Rev. D 86 P010001 (2012).

[3] Aaij R. et al. (LHCb collaboration), Measurements of CP violation in the three-body phase space of charmless $B^{ \pm}$decays, Phys. Rev. D 90112004 (2014) [hep-ex/1408.5373].

[4] CDF collaboration, Measurements of Direct CP-Violating Asymmetries in Charmless Decays of Bottom Baryons, Phys. Rev. Lett. 113242001 (2014) [hep-ex/1403.5586].

[5] Aaij R. et al. (LHCb collaboration), Searches for $\Lambda_{b}^{0}$ and $\Xi_{b}^{0}$ decays to $K_{\mathrm{S}}^{0} p \pi^{-}$and $K_{\mathrm{S}}^{0} p K^{-}$final states with first observation of the $\Lambda_{b}^{0} \rightarrow K_{\mathrm{S}}^{0} p \pi^{-}$decay, JHEP 04087 (2014) [hep-ex/1402.0770].

[6] Aaij R. et al., Search for the $\Lambda_{b}^{0} \rightarrow \Lambda \eta^{\prime}$ and $\Lambda_{b}^{0} \rightarrow \Lambda \eta$ decays with the LHCb detector, JHEP 09006 (2015) [hep-ex/1505.03295].

[7] Aaij R. et al. (LHCb collaboration), Observations of $\Lambda_{b}^{0} \rightarrow \Lambda K^{+} \pi^{-}$and $\Lambda_{b}^{0} \rightarrow \Lambda K^{+} K^{-}$decays and searches for other $\Lambda_{b}^{0}$ and $\Xi_{b}^{0}$ decays to $\Lambda h^{+} h^{(\prime)}$ - final states, JHEP 05081 (2016) [hep-ex/1603.00413].

[8] Aaij R. et al. (LHCb collaboration), Observation of the $\Lambda_{b}^{0} \rightarrow \Lambda \phi$ decay, Phys. Lett. B 759 282-292 (2016) [hep-ex/1603.02870].

[9] Aaij R. et al. (LHCb collaboration), Study of the kinematic dependences of $\Lambda_{b}^{0}$ production in $p p$ collisions and a measurement of the $\Lambda_{b}^{0} \rightarrow \Lambda_{c}^{+} \pi^{-}$branching fraction, JHEP 08143 (2014) [hep-ex/1405.6842].

[10] Leitner O., Ajaltouni Z.J., Testing CP and Time Reversal Symmetries with $\Lambda_{b}^{0} \rightarrow \Lambda V(1-)$ Decays, Nucl. Phys. Proc. Suppl. 174169 (2007) [hep-ph/0610189].

[11] Aaij R. et al. (LHCb collaboration), First observation of the decay $B_{s}^{0} \rightarrow K_{\mathrm{S}}^{0} K^{*}(892)^{0}$, JHEP 01012 (2016) [hep-ex/1506.08634]. 